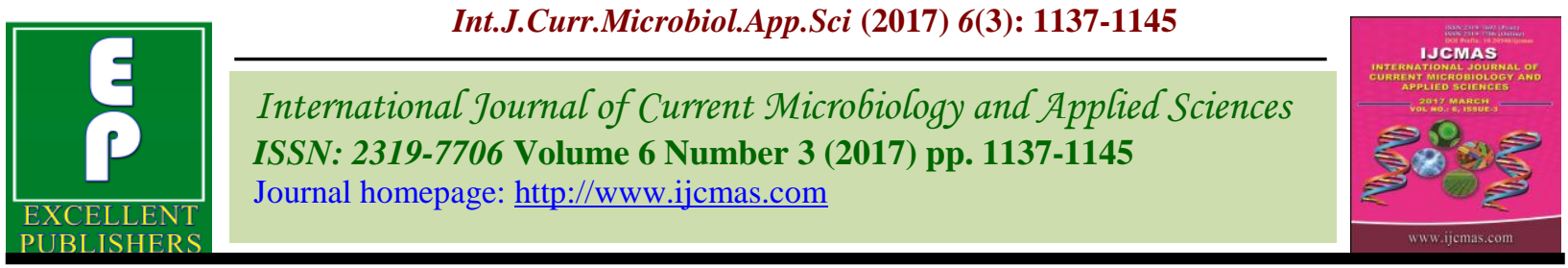

Original Research Article https://doi.org/10.20546/ijcmas.2017.603.132

\title{
Microbiological Profile of Blood Stream Infection in Neutropenic Patient in a Tertiary Care Centre
}

\author{
Joydeep Mangaraj* and Dipa Barkataki
}

\author{
Department of Microbiology, Gauhati Medical College, Assam, India \\ *Corresponding author
}

\section{A B S T R A C T}

Blood stream infection is a major cause of morbidity and mortality among neutropenic patients. Despite use of antibiotics and antifungals FN remains a therapeutic challenge. It prolongs hospital stay, increases health-care costs, and compromises chemotherapy efficacy in patients with malignancy who commonly experience neutropenia. This study depicts the microbiological profile, antibiotic sensitivity pattern and relation of BSI and

\begin{tabular}{|l|}
\hline Ke y w or d s \\
Blood Stream \\
Infection, \\
Neutropenic \\
Patient.
\end{tabular}
degree of neutropenia in the neutropenic patients. The study was a hospital based prospective observational study conducted in a tertiary care centre in North east, India. Correlation of degree of neutropenia and BSI with analysis of microbiological profile and antibiotic sensitivity pattern of the FN patients admitted from September 2014 to September 2016 was done. A total of 259 cases of fever with neutropenia were included from September 2014 to September 2016 in the present study. Of these, $55(21.33 \%)$ were found to be culture positive. Majority of case 79 (30.5\%) were suffering from Acute Myeloid Leukemia (AML). Gram negative bacilli $58.18 \%$ (32) is the most common isolate. The antibiotic sensitivity among GNB was highest for colistin (100\%) and teigecycline (93.8\%). There is an alarming increase in resistance for cephalosporins and Carbapenems. The frequency of isolation of micro organisms in blood by blood culture in neutropenic patient with fever is $21.23 \%$ and Gram negative bacilli is still the predominant pathogens. There is an alarming rise in the resistance against carbapenems and commonly used drugs. It needs to be emphasized that a routine microbiological examination of these group of patients should be carried out routinely and periodically so as to analyse and compare the changing trends in the microbial aetiology and the antibiogram patterns. It is recommended that an effective empirical antibiotic regime could be tailored for this group of patients to decrease the morbidity and mortality.

\section{Introduction}

Blood stream infection is a major cause of morbidity and mortality among neutropenic patients (Tariq et al., 2002; Honar et al., 2003). Despite use of antibiotics and antifungals FN remains a therapeutic challenge. It increases health-care costs, prolongs hospital stay and compromises chemotherapy efficacy, due to delays and dose reductions. Susceptibility to infectious diseases increases sharply when neutrophil counts fall below 1000 cells $/ \mu \mathrm{L}$. Acute neutropenia, such as that caused by cancer chemotherapy, is more likely to be associated with increased risk of infection than neutropenia of long duration (months to years) that reverses in response to infection or carefully controlled administration of endotoxin (Steven et al.,). 
In about $20-30 \%$ of the episodes of neutropenic fever, bacteremia is diagnosed. The crude rate for blood stream infections (BSI) in cancer patients ranges from 18 to 42\% (Ehni et al., 1991; Prabhash et al.,). While the incidence of gram-negative infections $(71 \%)$ was higher than grampositive (29\%) in the 1960s and 1970s, increased use of indwelling catheters, earlygeneration quinolone prophylaxis, and broadspectrum empirical anti gram-negative antibacterial therapy led to an increase in the incidence of gram-positive pathogens (69\%) in the 1980s and 1990s (Alison et al., 2010; Schimpif, 1991; Reuben et al., 2004). Honar Cherif et al., (2003) in their 14 years study found Coagulase Negative Staphylococci (17\%) being the dominating pathogen. Akihisa kanamaru et al., (2004) found Gram negative organisms were more prevalent during 1985-1996, with pseudomonas species being the commonest but Gram positive organisms were more prevalent, with staphylococcus species dominance during 1997-2002. Anaerobes are infrequent cause. In an Asian study by Tariq Butt et al., no anaerobic organisms were isolated.

Febrile neutropenia $(\mathrm{FN})$ is one of the major causes of morbidity and mortality in patients of hematological malignancies. These patients may not present with any obvious source of infection except blood stream infection. Hence, in all patients suspected of FN, blood culture sensitivity should be sent if possible before the first dose of antibiotics. These patients generally have associated anaemia and thrombocytopenia (Arun et al., 2013).

There has been a considerable change in spectrum of pathogens causing infection in neutropenic patients. Prabash et al., in a retrospective study, in India in the year 2007 found high degree of resistance to the cephalosporins with only $27.1 \%$ of the Gram Negative isolates being sensitive to third generation cephalosporins, namely ceftriaxone and cefotaxime. Meropenem was the most effective antibiotic, active against $71.7 \%$ of the Gram negative isolates. The antibiotic most active against Gram positive was linezolid. Because the progression of infection in neutropenic patients can be rapid, and because such patients cannot be reliably distinguished from non-infected patients upon presentation, empirical antibiotic therapy should be administered promptly to all neutropenic patient at the onset of fever. It is administered with the goal of eradicating the most frequent organisms causing fulminant infections, which may result in serious complications. In the setting of changing flora and susceptibility patterns to antibiotics, guidelines as to "best therapy" of infection in the neutropenic patient must be evaluated on the basis of local patterns of infection and local and regional resistance patterns

So there is a need to know the local pattern of organisms causing bacteremia, so that an effective empirical antibiotic therapy can be formulated for the neutropenic patients to reduce the mortality and morbidity rate. Keeping this in view, the present study was carried out in a tertiary care centre to know the frequency of bacteremia in neutropenic patient, to isolate the organisms and study the antibiotic sensitivity pattern causing bacteremia in neutropenic patients.

\section{Materials and Methods}

The study was a hospital based prospective observational study conducted in a tertiary care centre. Analysis of microbiological profile and Antibiotic sensitivity pattern of the FN patients admitted from September 2014 to September 2016.

259 cases presenting with Fever and Absolute neutrophil count $<500 / \mathrm{mm}^{3}$ (Fever was defined as single oral temperature of $\geq 38.3^{0}$ 
Celsius $\left(101^{0} \mathrm{~F}\right)$ or a temperature of $\geq 38^{0}$ Celsius $\left(100.4^{0} \mathrm{C}\right)$ for more than 1 hour were included in the study. Patients who became febrile in proximity to having received blood products and Patients not willing to undergo the procedure were excluded from the study.

History was obtained and physical examination was performed. Blood was drawn for blood culture before empirical antimicrobial therapy was started or just before the next dose of antibiotic. One specimen of blood for aerobic and anaerobic cultures was drawn through peripheral vein. A second sample was collected at the same day from a different venipuncture to rule out contamination with skin flora.

Immediately after inoculation, VersaTREK Blood culture (Redox1, Redox2) bottles were incubated in the VersaTrek. Subculture was done from the incubated broths on $10 \%$ sheep Blood Agar, MacConkey's Agar and Chocolate Agar when flagged positive. A smear was also made from the broth after inoculation and gram stain was done. The smear was examined for presence of any microorganisms.

The inoculated Blood Agar and MacConkey's Agar media plates were incubated at $37^{\circ} \mathrm{C}$ for 24 hours aerobically. The inoculated Chocolate Agar plates were kept in Oxoid Anaerobic jar with the anaerobic gas pack (HIMEDIA) and anaerobic indicator, and incubated at $37^{\circ} \mathrm{C}$ for 24 hours. Characterization and identification of organisms was done as per Collee et al., (1996).

Biochemical identifications of the isolates were carried out by conventional methods and VITEK 2 compact system.

All the isolated bacteria were tested against different antimicrobial agents by the Vitek2 as well as the standard disc diffusion method (Kirby Bauer Technique). Commercially available AST kits AST-P628 (REF 414534) for Gram Positive cocci and AST-N281 (REF 414532) for Gram Negative bacilli were used from biomerieux. For disc diffusion method commercially available antibiotic discs were used obtained from Hi Media Laboratories Limited.

\section{Results and Discussion}

A total of 259 cases of fever with neutropenia were included from September 2014 to September 2016 in the present study. Blood samples were collected from the various department and Intensive care units (ICU) of Gauhati medical college. Bacteriological studies of the specimens were carried out in the department of microbiology, Gauhati Medical College and Hospital for a period of 2 years. Blood cultures from neutropenic patients with fever were collected and processed, with maximum patients from Hematology department 189 (73\%) and M: F ratio $1.7: 1$. Of these, $55(21.33 \%)$ were found to be culture positive. Highest number of cases was in the younger age group.

Majority of case 79 (30.5\%) were suffering from Acute Myeloid Leukemia (AML), followed by 73 (28.2\%) Acute Lymphocytic Leukemia (ALL) (Table 1).Table 2 indicates that the occurrence of BSI is directly proportional to the Absolute Neutrophil count, with highest 15 (88.23\%) positive Blood culture in patients with ANC $<100 / \mu 1$. Out of the 259 neutropenic patients only 53(21.23\%) were blood culture positive. However no anaerobic organisms were isolated. 2 isolates of Candida tropicalis were isolated (Table 3). Gram negative bacilli $58.18 \%$ (32) is the most common isolate followed by $38.18 \%$ (21) Gram positive cocci (Table 4). 
Among the Gram negative isolates Escherichia coli $50 \%$ (16) is the predominant isolate followed by Klebsiella pneumoniae $43.8 \%$ (14) and Acinetobacter baumannii complex $6.25 \%$ (2) (Table 5). A total of $38.18 \%$ (21) Gram positive cocci were identified of which $47.61 \%$ (10) were Coagulase negative Staphylococci (CONS), the predominant organism isolated among GPC, $28.57 \%$ (6) were Staphylococcus aureus, and 5 (23.8\%) were of Enterococcus species (Table 6). 2 MRSA was also detected among the isolates.

The antibiotic sensitivity among GNB was highest for Colistin (100\%) and Teigecycline $(93.8 \%)$. There is an alarming increase in resistance for cephalosporins and carbapenems (Table 7). The GPC were sensitive to Daptomycin, Teigecycline, Vancomycin, Teicoplanin and Linezolid. However they were highly resistant to Fluroquinolones and Clindamycin (Table 8 and Fig. 1).

In our study we observed that the Gram positive isolates were $100 \%$ sensitive to Teigecycline and Daptomycin followed by Linezold, Vancomycin and Teicoplanin with $90.5 \%$ sensitive. Whereas clindamycin was $100 \%$ resistant followed by ciprofloxacin, levofloxacin and erythromycin with 66.7, $66.7 \%$ and $76.2 \%$ resistance respectively. MRCoNS are a concerning issue as they are resistant to many drugs.

Patients with malignancies and neutropenia are at high risk for the development of Blood stream infection (BSI). The causative organisms have changed over time. The Gram-negative bacteria were the main responsible for the febrile neutropenia in the sixties; their impact declined due to the use of fluoroquinolone prophylaxis. This situation was followed by the gradual emergence of Gram-positive bacteria also following the increased use of intravascular devices and the introduction of new chemotherapeutic strategies. In the last decade, the Gramnegative etiology is raising again because of the emergence of resistant strains that make questionable the usefulness of current strategies for prophylaxis and empirical treatment.

This necessitates a periodic monitoring of the locally prevalent pathogens and their antibiograms, so that a rational empiric antimicrobial therapy for neutropenic patients may be formulated.

In the present study, out of 259 cases, highest number of case $73 \%$ were from Haematology department and majority of cases $30.5 \%$ were suffering from Acute Myeloid Leukemia (AML), followed by $28.2 \%$ Acute Lymphocytic Leukemia (ALL). Similar results were obtained in other studies, Michelle Karim et al., (2015) with $42 \%$ cases having AML, followed by $12 \%$ ALL. Lakshmaiah et al., (2015) 2011 - 2013, also reported AML (55 episodes) as the most common etiology. The high incidence of incidence of febrile neutropenia in AML could be due to the use of intensive chemotherapy leading to prolonged and profound neutropenia, thus increasing the risk of infection.

Our study reveals that the rate of blood culture positivity is highest $(88.23 \%)$ in those patients with ANC <100/ $\mu$ l and only $13.04 \%$ in ANC $>200 / \mu 1$. Similar relation was established by Bodey et al., (1966) according to the study the frequency of severe infection is highest when absolute neutrophil count was $100 / \mu 1$ and proportionately less frequent at ANC 100 $-500 / \mu \mathrm{l}$ and ANC 500-1000/ $\mu 1$. Only $21.23 \%$ (53) has shown the growth in blood culture. Similar results were reported by Lakshmaiah et al., 2011 - 2013, in South India $19.44 \%$ positive blood culture in neutropenic patients. But in a study Mandal et 
al., (2015) 2010-2013 in a similar study in India found $29.1 \%$ culture positivity. However no anaerobic organisms were isolated in our study, which is in conformity with other similar studies done by Tariq Butt et al., 2002. However Honar Cherif et al., 1988-2001 found 4.1\% anaerobic isolates. The positivity rate can be probably explained by the observation made by Matsuhisa et al., (1994) they observed in febrile neutropenic patients most bacteria are phagocytized by neutrophils and only few remain in blood to form colonies detectable by culture thus emphasizing the use of in situ hybridization to detect bacterial DNA.

Our study shows Gram negative bacilli $58.18 \%$ (32) is the most common isolate followed by $38.18 \%$ (21) Gram positive cocci. Similar observation was made by other workers in India, Lakshmaiah et al., 2011 2013, reported, GNB $57.14 \%$ (12) as predominant isolate and GPC 42.86\% (9). Mandal et al., (1994) 2010-2013 reported
GNB $61.53 \%$ (48) as the predominant isolate followed by GPC $34.61 \%$ (27). Dong -Gun Lee et al., (2011) mentioned that as a general characteristic of Asia-Pacific region, Gramnegative microorganisms were the major pathogens of infection.

Among the 32 Gram negative isolates Escherichia coli 50\% (16) is the predominant isolate followed by Klebsiella pneumoniae 43.75\% (14) and Acinetobacter baumannii complex 6.3\% (2). Tariq Butt et al., 2002, reported $13.25 \%$ (11) E. coli as the predominant isolate. A total of $38.18 \%$ (21) Gram positive isolates were identified of which $47.61 \%$ (10) were coagulase negative Staphylococci, the predominant organism, $28.57 \%$ (6) were Staphylococcus aureus, and $23.8 \%$ (5) were of Enterococcus species isolated among the GPC. Similar results were seen by Tariq Butt et al., (2002), most common isolates were CONS 26.5\% (22), Prabhash et al., (2007), CONS 10.54\% (51) among GPC isolates.

Table.1 Distribution of total diagnosed cases

\begin{tabular}{|c|c|c|}
\hline Diagnosis & No. & Percentage \\
\hline Solid tumors & 21 & 8.1 \\
\hline Acute Myeloid Leukemia & 79 & 30.5 \\
\hline Acute Lymhocytic Leukemia & 73 & 28.2 \\
\hline $\begin{array}{c}\text { Chronic Lymhocytic } \\
\text { Leukemia }\end{array}$ & 5 & 1.9 \\
\hline Chronic Myeloid Leukemia & 15 & 5.8 \\
\hline Non - Hodgkin's Lymphoma & 13 & 5 \\
\hline Hodgkin's Lymphoma & 3 & 1.2 \\
\hline Myelo Dysplastic Syndrome & 8 & 3.1 \\
\hline Acute leukemia & 21 & 8.1 \\
\hline Aplastic anaemia & 10 & 3.9 \\
\hline Hypoplastic anaemia & 3 & 1.2 \\
\hline Multiple myeloma & 5 & 1.9 \\
\hline Langerhan's cell histocytosis & 3 & 1.2 \\
\hline
\end{tabular}


Table.2 Relation of ANC and culture positive

\begin{tabular}{|l|l|l|}
\hline ANC $(/ \boldsymbol{\mu l})$ & $\begin{array}{l}\text { No. of FN } \\
\text { episodes }\end{array}$ & $\begin{array}{l}\text { NO.(\%) of positive } \\
\text { Blood culture }\end{array}$ \\
\hline$<100$ & 17 & $15(88.23 \%)$ \\
\hline $100-200$ & 35 & $13(37.14 \%)$ \\
\hline $201-500$ & 207 & $27(13.04 \%)$ \\
\hline
\end{tabular}

Table.3 Total blood culture positive cases

\begin{tabular}{|r|l|l|l|}
\hline $\begin{array}{r}\text { Total no. of } \\
\text { Blood Culture }\end{array}$ & \multicolumn{2}{|l|}{ No. of culture positive cases } & percentage \\
\hline \multirow{2}{*}{259} & Aerobic Bacteria & 53 & $20.46 \%$ \\
\cline { 2 - 4 } & $\begin{array}{l}\text { Anaerobic } \\
\text { Bacteria }\end{array}$ & 0 & $0 \%$ \\
\cline { 2 - 4 } & Fungus & 02 & $0.77 \%$ \\
\hline
\end{tabular}

Table.4 Isolates in total positive blood culture

\begin{tabular}{|l|l|l|}
\hline Pathogens & $\begin{array}{l}\text { Total No. } \\
\text { of isolates }\end{array}$ & percentage \\
\hline GPC & 21 & $38.18 \%$ \\
\hline GNB & 32 & $58.18 \%$ \\
\hline Fungus & 2 & $3.64 \%$ \\
\hline Total & 55 & $100 \%$ \\
\hline
\end{tabular}

Table.5 Different isolates of Gram negative organisms

\begin{tabular}{|l|l|l|}
\hline Gram Negative Bacilli & No. & Percentage \\
\hline Escherichia coli & 16 & $50 \%$ \\
\hline Klebsiella pneumoniae & 14 & $43.8 \%$ \\
\hline Acinetobacter Spp. & 2 & $6.25 \%$ \\
\hline Total isolates & 32 & $100 \%$ \\
\hline
\end{tabular}

Table.6 Different isolates of Gram positive cocci

\begin{tabular}{|l|l|l|l|}
\hline Gram positive cocci & \multicolumn{2}{|l|}{ No. of isolates } \\
\hline Staphylococcus aureus & \multicolumn{2}{|c|}{$6(28.57 \%)$} \\
\hline $\begin{array}{l}\text { Coagulase } \\
\text { Negative } \\
\text { Staphylococci }\end{array}$ & Staphylococcus hemolyticus & 6 & \multirow{2}{*}{$10(47.6 \%)$} \\
\cline { 2 - 3 } Enterococci & Staphylococcus saprophyticus & 4 & \multirow{2}{|c|}{$21(23.8 \%)$} \\
\cline { 2 - 3 } & Enterococcus fecalis & 4 & \\
\cline { 2 - 4 } Total cases & Enterococcus faecium & 1 & \multicolumn{2}{|c|}{} \\
\hline
\end{tabular}


Table.7 Sensitivity pattern of GNB isolates

\begin{tabular}{|l|l|l|l|}
\hline ISOLATES & MDR\% & PDR\% & VA Resistance\% \\
\hline Staphylococcus aureus & 16.7 & 0 & 33.3 \\
\hline CONS & 20 & 0 & 0 \\
\hline Enterococci & 0 & 0 & 0 \\
\hline
\end{tabular}

Table.8 Sensitivity of gpc isolates

\begin{tabular}{|c|c|c|c|c|c|c|c|c|c|c|c|c|c|c|c|c|c|}
\hline $\begin{array}{l}\text { Isolates } \\
\text { of GPC }\end{array}$ & & CX & $\mathbf{P}$ & $\overline{\mathbf{O X}}$ & $\begin{array}{l}\text { GE } \\
\mathbf{N} \\
\end{array}$ & CIP & $\begin{array}{l}\text { LEV } \\
\text { O }\end{array}$ & CD & $\mathbf{E}$ & $\mathbf{L Z}$ & DAP & TEI & $\overline{\text { VA }}$ & TET & $\begin{array}{l}\text { TG } \\
\text { C }\end{array}$ & RIF & $\begin{array}{l}\mathrm{CO} \\
\mathrm{T} \\
\end{array}$ \\
\hline \multirow{2}{*}{$\begin{array}{l}\text { Staphylo } \\
\text { coccus } \\
\text { aureus } \\
\mathrm{n}=6\end{array}$} & No. & 4 & 2 & 4 & 4 & 2 & 2 & 0 & 2 & 6 & 6 & 4 & 4 & 6 & 6 & 4 & 4 \\
\hline & $\%$ & $\begin{array}{c}66 . \\
7\end{array}$ & $\begin{array}{c}33 . \\
3\end{array}$ & 66.7 & 67 & 33 & 33.3 & 0 & 33 & 100 & 100 & 67 & 66.7 & 100 & 100 & 66.7 & 66.7 \\
\hline \multirow{2}{*}{$\begin{array}{l}\text { CONS } \\
\mathrm{n}=10\end{array}$} & No. & 0 & 0 & 0 & 10 & 5 & 5 & 0 & 0 & 10 & 10 & 10 & 10 & 5 & 10 & 3 & 10 \\
\hline & $\%$ & 0 & 0 & 0 & 100 & 50 & 50 & 0 & 0 & 100 & 100 & 100 & 100 & 50 & 100 & 30 & 100 \\
\hline \multirow{2}{*}{$\begin{array}{l}\text { Entero } \\
\text { coccus } \\
n=5\end{array}$} & No. & & 0 & & 3 & 0 & 0 & & 3 & 3 & 5 & 5 & 5 & 0 & 5 & & \\
\hline & $\%$ & & 0 & & 60 & 0 & 0 & & 60 & 60 & 100 & 100 & 100 & 0 & 100 & & \\
\hline \multirow[t]{2}{*}{$\begin{array}{l}\text { Total } \\
\mathrm{n}=21\end{array}$} & No. & & & & 17 & 7 & 7 & 0 & 5 & 19 & 21 & 19 & 19 & 11 & 21 & $\begin{array}{c}7 \\
(n=1 \\
6)\end{array}$ & $\begin{array}{c}14 \\
(\mathrm{n}=1 \\
6)\end{array}$ \\
\hline & $\%$ & & & & 81 & 33.3 & 33.3 & 0 & $\begin{array}{c}23 . \\
8\end{array}$ & $\begin{array}{c}90 . \\
5\end{array}$ & 100 & 90.5 & 90.5 & 55.6 & 100 & 43.8 & 87.5 \\
\hline
\end{tabular}

$\mathrm{P}=$ penicillin, $\mathrm{OX}=$ Oxacillin, $\mathrm{E}=$ Erythromycin, GEN= Gentamicin, LEVO= Levofloxacin, $\mathrm{CD}=$ Clindamycin, LZ= Linezolid, DAP=

Daptomycin, TEI $=$ Teicoplanin, $\mathrm{VA}=$ Vancomycin, $\mathrm{TET}=$ Tetracycline, $\mathrm{TGC}=$ Teigecycline, $\mathrm{RIF}=\mathrm{Rifampicin}, \mathrm{COT}=\mathrm{Cotrimoxazole}, \mathrm{CX}=$ Cefoxitin

Fig.1 Sensitivity pattern of GNB isolates

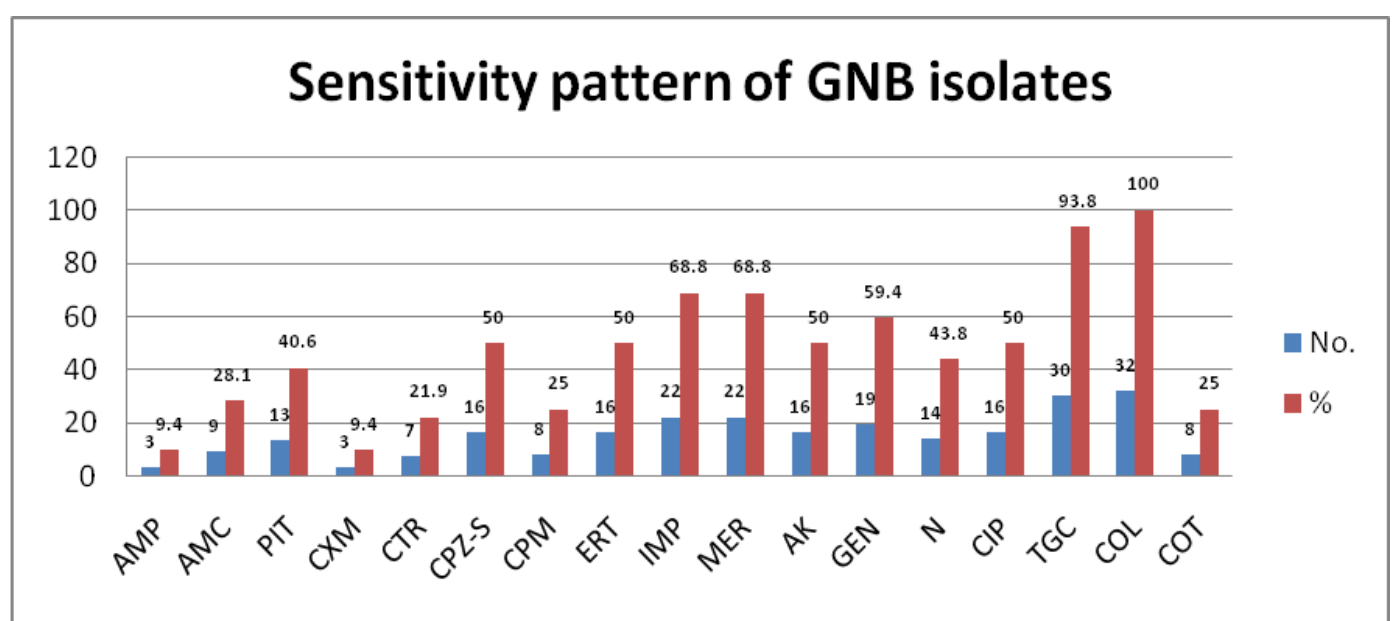

$\mathrm{AMP}=$ Ampicillin, $\mathrm{AMC}=$ Amoxycillin + clavulinic acid, $\mathrm{PIT}=$ Piperacillin + Tazobactam $, \mathrm{CXM}=\mathrm{Cefuroxime}, \mathrm{CTR}=\mathrm{Ceftriaxone}, \mathrm{CPZ}-$ $\mathrm{S}=$ Cefoperazone + sulbactam, $\mathrm{CPM}=$ Cefepime, ERT=Ertapenem, IMP=Imipenem, MER=Meropenem, Ak=amikacin, GEN=Gentamicin, N= Nalidixic acid, CIP= Ciprofloxacin, TGC=Teigecycline, $\mathrm{COL}=$ Colistin, $\mathrm{COT}=$ Cotrimoxazole

In this study we observed that the 32 Gram negative bacilli isolated were $100 \%$ sensitive to Colistin, followed by $93.8 \%$ sensitive to Teigecycline and $68.8 \%$ sensitive to Imipenem and Meropenem. However contrasting our findings
Kuntegowdanahalli C Lakshmaiah et al., 20112013, found imipenem 100\% sensitive, followed by Pipercillin-tazoactum $86.95 \%$ sensitive. Tariq Butt et al., 2002, also found imipenem to be $100 \%$ sensitive. 
In this study it was observed that the Gram positive isolates were $100 \%$ sensitive to teigecycline and daptomycin followed by linezold, vancomycin and teicoplanin with $90.5 \%$ sensitive. Whereas clindamycin was $100 \%$ resistant, the antimicrobial susceptibility pattern found is similar to other workers, Tariq Butt et al., 2002 reported vancomycin and Imipenem to be $100 \%$ sensitive and Ampicillin to be $88.9 \%$ resistant. Prabhash et al., 2007, Linezolid was $100 \%$ sensitive.

In conclusion, the findings of the present study revealed that the frequency of isolation of bacteria in blood by blood culture in neutropenic patient with fever is $21.23 \%$ and Gram negative bacilli is still the predominant pathogens.

The antibiograms revealed that the Gram positive cocci were $100 \%$ sensitive to teigecycline and daptomycin but resistance to commonly used drugs are at a rise.

The Gram negative bacilli isolated were $100 \%$ sensitive to colistin and $93.8 \%$ to teigecycline, but there is an alarming rise in the resistance against carbapenems and commonly used drugs.

As the data of antibiogram of the isolates from neutropenic patients is sparse in this region and as seen in our study a fall in neutrophil count $<100$ makes the individual vulnerable to infection by microorganisms so it needs to be emphasized that a routine microbiological examination of these group of patients should be carried out routinely and periodically so as to analyse and compare the changing trends in the microbial aetiology and the antibiogram patterns. So that an effective empirical antibiotic regime could be tailored for this group of patients to decrease the morbidity and mortality.

Financial support: DBT MD/MS Thesis Grant Scheme, Tezpur University

\section{References}

Akihisa Kanamaru and Youichi Tatsumi. 2004. Microbiological Data for Patients with Febrile Neutropenia. Clin. Infect. Dis., 39:
S7-10 2004 by the Infectious Diseases Society of America.

Alison, G., Freifeld, Eric, J., Bow, Kent, A. Sepkowitz, Michael, J. Boeckh, James I. Ito, Craig A. Mullen, Issam I. Raad, Kenneth V. Rolston, Jo-Anne H. Young, and John, R. Wingard. 2010. Clinical Practice Guideline for the Use of Antimicrobial Agents in Neutropenic Patients with Cancer: 2010 Update by the Infectious Diseases Society of America

Arun, B., Karanwal, Bharat, J., Parikh, Parijat Goswami, Harsha, P., Panchal, Bhavesh B. Parekh, Kaushal, B. Patel. 2013. Review of clinical profile and bacterial spectrum and sensitivity patterns of pathogens in febrile neutropenic patients in hematological malignancies: A retrospective analysis from a single center. Indian J. Med. Paediatr. Oncol., 34(2): 85-88.

Bodey, G.P., Buckley, M., Sathe, Y.S., et al. 1966. Quantitative relationships between circulating leukocytes and infection in patients with leukemia. Ann. Intern. Med., 64: 328-340.

Collee, J.G., Miles, R.S., Watt, B. 1996. Test for identification of bacteria. In:Mackie and McCartney Practical Medical Microbiology. Collee JG, Fraser AG, Marmion BP, Simmons AC(editor), $14^{\text {th }}$ edition. Churchill Livingstone: Newyork; 131-149.

Ehni, W.F., Reller, L.B., Ellison, R.T. 1991. 3rdBacteremia in granulocytopenic patients in a tertiary-care general hospital. Rev. Infect. Dis., 13(4): 613-9.

Gudiol, C., Bodro, M., Simonetti, A., Tubau, F., González-Barca, E., Cisnal, M., DomingoDomenech, E., Jiménez, L., Carratalà, J. Changing aetiology, clinical features, antimicrobial resistance, and outcomes of bloodstream infection in neutropenic cancer patients. Clin. Microbiol. Infect., 19: 474-9. PMid: 22524597

Honar Cherif, Goran Kronvall, Magnus Bjo“rkholm and Mats Kalin. 2003. Bacteraemia in hospitalised patients with malignant blood disorders: a retrospective study of causative agents and their resistance profiles during a 14-year period 
without antibacterial prophylaxis. The Hematol. J., 4; 420-426.

Klastersky, J.2004. Management of fever in neutropenic patients with different risks of complications. Clin. Infect. Dis., 39(Suppl 1): S32-7.

Kuntegowdanahalli, $\quad$ C., Lakshmaiah, Abhayakumar, S., Malabagi, Govindbabu, Rachan Shetty, Mahua Sinha, Rudrapatna, S. Jayashree. Febrile Neutropenia in Hematological Malignancies: Clinical and Microbiological Profile and Outcome in High Risk Patients; J. Lab. Physicians, Vol-7 / Issue-2.

Lee, D.G., Kim, S.H., Kim, S.Y., Kim, C.J., Park, W.B., Song, Y.G., et al. 2011. Evidencebased guidelines for empirical therapy of neutropenic fever in Korea. Korean $J$. Intern. Med., 26(2): 220-52. [DOI] [PubMed](cross-ref)

Matsuhisa, A., Saito, Y., Ueyama, H., et al. 1994. Detection of bacteria phagocyte smears from septicemia-suspected blood by in situ hybridization using biotinylated probes. Microbiol. Immunol., 38: 511-7.

Michelle Karim, Waheed Khan, Imtiaz Malik, Badar Farooqi. 1991. Bacterial isolates in neutropenic febrile patients; J. Pak. Med. Assoc., p-35-37

Mikulska, M., Viscoli, C., Orasch, C., Livermore, D.M., Averbuch, D., Cordonnier, C., Akova, M. 2013. Fourth European Conference on Infections in Leukemia Group (ECIL-4), a joint venture of EBMT, EORTC, ICHS, ELN and ESGICH/ESCMID. Aetiology and resistance in bacteraemias among adult and paediatric haematology and cancer patients. J. Infect., 68: 321-31.

Prabhash, K., A. Medhekar, N. Ghadyalpatil, V. Noronha, S. Biswas, P. Kurkure, R. Nair, R. Kelkar. Blood stream infections in cancer patients: A single center experience of isolates and sensitivity pattern. Indian J. Cancer.

Prakas Kumar Mandal, Suman Kumar Maji, Tuphan Kanti Dolai,Rajib De, Shyamali Dutta,Sandeep Saha, Maitreyee Bhattacharyya. 2015. Micro-organisms Associated with Febrile Neutropenia in Patients with Haematological Malignancies in a Tertiary Care Hospital in Eastern India. Indian J. Hematol. Blood Transfusion, Volume 31, Issue 1, pp 46-50.

Reuben Ramphal. 2004. Changes in the Etiology of Bacteremia in Febrile Neutropenic Patients and the Susceptibilities of the Currently Isolated Pathogens. Clin. Infect. Dis., 39(Supplement 1): S25 S31.

Sara Lo Menzo, Giulia la Martire, Giancarlo Ceccarelli and Mario Venditti. 2015. New insight on epidemiology and management of bacterial bloodstream infection in patients with hematological malignancies. Mediterr. J. Hematol. Infect. Dis., 7(1): e2015044.

Schimpif, S.C. "Infections in patients with cancer: overview and epidemiology" Comprehensive test on oncology; 2: 172032.

Steven, M., Holland, John, Gallin. Disorders of Granulocytes and Monocytes Principles of internal medicine Harrison's $19^{\text {th }}$ edition, page no. 417.

Tariq Butt, Raja Kamran Afzal, Rifat Nadeem Ahmad, Muhammad Salman, Abid Mahmood, Masood Anwar. 2002. Bloodstream infections in febrile neutropenic patients: bacterial spectrum and antimicrobial susceptibility pattern. J. Ayub Med. Coll., Abbottabad: JAMC, 16(1):1822

Zinner, S.H. 1999. Changing epidemiology of infections in patients with neutropenia and cancer: emphasis on gram-positive and resistant bacteria. Clin. Infect. Dis., 29: 490-4.

\section{How to cite this article:}

Joydeep Mangaraj, Dipa Barkataki. 2017. Microbiological Profile of Blood Stream Infection in Neutropenic Patient in a Tertiary Care Centre. Int.J.Curr.Microbiol.App.Sci. 6(3): 1137-1145. doi: https://doi.org/10.20546/ijcmas.2017.603.132 